\title{
Impact of Residual Adenopathy Following Chemoradiotherapy for Squamous Cell Carcinoma of the Head and Neck: A Retrospect ve Analysis of 51 Consecut ve Cases
}

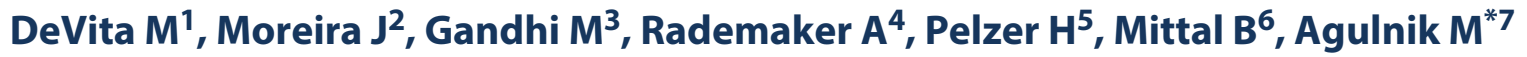 \\ ${ }^{1}$ Department of Medicine, Northwestern University Feinberg School of Medicine, USA \\ ${ }^{2}$ Division of Hematology/Oncology, Department of Medicine, Northwestern University Feinberg School of Medicine, USA \\ ${ }^{3}$ Division of Hematology/Oncology, Department of Medicine, Northwestern University Feinberg School of Medicine, USA \\ ${ }^{4}$ Professor of Preventive Medicine, Directory, Biostatistics Collaboration Center, USA
}

${ }^{5}$ Associate Professor of Otolaryngology, Department of Otolaryngology, Northwestern University, Feinberg School of Medicine, USA

${ }^{6}$ Professor of Radiation Oncology, Department of Radiation Oncology, Robert H. Lurie Comprehensive Cancer Center, Northwestern University, Feinberg School of Medicine, USA

${ }^{7}$ Associate Professor of Medicine, Division of Hematology \& Oncology, Robert H Lurie Comprehensive Cancer Center, Northwestern University Feinberg School of Medicine, USA

${ }^{*}$ Corresponding author: Agulnik M, Division of Hematology and Oncology, Robert $\mathrm{H}$ Lurie Comprehensive Cancer Center, Feinberg School of Medicine, Northwestern University, Chicago, USA, Tel: 312-695-6180; E-mail: m-agulnik@northwestern.edu

Received date: Apr 06, 2016; Accepted date: May 06, 2016; Published date: May 13, 2016

Copyright: (c) 2016 Agulnik M, et al. This is an open-access article distributed under the terms of the Creative Commons Attribution License, which permits unrestricted use, distribution, and reproduction in any medium, provided the original author and source are credited.

\section{Abstract}

Locally advanced squamous cell carcinoma of the head and neck is commonly treated with chemoradiotherapy (CRT). It remains uncertain if residual adenopathy (RA), greater than $1 \mathrm{~cm}$ in maximal dimension, detected by computerized tomography following treatment has an impact on outcome. Similarly, the utility of neck dissection (ND) to pathologically evaluate RA remains undefined. We performed a retrospective analysis to assess the frequency and impact of RA and the utility of ND in SCCHN.

A query of the electronic medical record using ICD9 codes was performed to identify patients that met the following inclusion criteria: age greater than 18 years, a histologically proven SCCHN diagnosed between 2003 and 2013, N1 to N3 disease, and treatment with CRT. Clinical data were abstracted through chart review. Progression free (PFS) and overall survival (OS) were estimated using Kaplan Meier analysis, and comparisons were made with log rank test. Chi-square and receiver operating characteristics were used to assess size of RA as a predictive factor.

One hundred twenty three patient charts were reviewed and ninety-nine complete patient records were available for analysis. The median age at diagnosis was 56 years. Eight percent had N1 disease, $86 \%$ had N2, and $5 \%$ had N3. Following completion of CRT, 51 patients (51\%) had RA. Of these, 41 patients underwent a ND, and 8 (19.5\%) were found to have residual malignancy. At a median follow up of 35 months, there was no difference in PFS or OS in the patients who had radiological RA ( $p=0.37$ and 0.72 ) or pathologically proven residual disease compared to those who did not. Residual adenopathy was not a predictor of residual pathologic disease. Size of RA was a poor predictor of residual cancer ( $A \cup C=0.637$ ). Of the 48 patients tested for $\mathrm{p} 16,77 \%$ were positive by immunohistochemistry. Patients with $\mathrm{p} 16$ positive tumors appeared to have superior OS ( $p=0.038)$, but there was no impact on PFS $(p=0.27)$ compared to those that were negative.

Following CRT, while many patients have RA, only a small subset (19.5\%) has residual active cancer. The presence of either does not impact PFS or OS. Moreover, using RA greater than $1 \mathrm{~cm}$ as a threshold to perform an ND lacks specificity in identifying those with pathologically positive residual cancer. These data suggest ND may be safely avoided in most patients and will help inform future prospective trials using alternative imaging modalities, such as PET/CT, to identify the subset of patients most likely to benefit from ND.

Keywords: Adenopathy; Carcinoma; Chemotherapy; Radiation

\section{Introduction}

Squamous cell carcinoma of the head and neck (SCCHN) encompasses malignancies of the lips, oral cavity, pharynx, and larynx, which share common mechanisms of carcinogenesis and have a similar treatment paradigm. There are an 
estimated 53,000 new cases annually in the United States [1] with 11,000 anticipated deaths. While tobacco and alcohol use have traditionally been the predominant risk factors, there has been an epidemiological shift over the last two decades, as an increasing proportion of patients present with human papilloma virus (HPV) driven tumors $[2,3]$.

Primary surgical resection or radiation therapy remains the preferred therapy for early stage disease without cervical lymph node involvement, but treatment strategies have evolved for locally advanced disease. Surgical intervention is reserved for residual or refractory disease. Earlier studies demonstrated the benefit of sequential chemotherapy with radiation, compared to upfront surgery, for organ preservation and morbidity, without compromising clinical outcome $[4,5]$. Subsequent trials established the role of concurrent chemoradiotherapy (CRT) as frontline therapy, with superior five-year overall survival compared to sequential therapy [6]. Consensus guidelines now advocate platinum-based chemotherapy concurrent with radiation therapy, and reassessment with cross sectional imaging at completion of therapy [7]. Notably, several series have demonstrated superior outcomes with this approach among patients with HPV positive disease $[8,9]$.

The utility of neck dissection (excision and pathologic evaluation of residual disease evident on imaging) following completion of definitive CRT for locally advanced SCCHN remains uncertain. Studies have drawn mixed conclusions, without clear superiority of planned neck dissection, responsebased neck dissection, or observation [10,11]. Given theoretical concern for a persistent neoplastic process resistant to CRT, or subclinical metastasis, neck dissection (ND) remains an option in current guidelines. The definition of residual disease is not established. Institutionally, if there is lymphadenopathy greater than $1.0 \mathrm{~cm}$ in diameter identified on post-treatment computerized tomography (CT), a ND is considered. ND has the potential to cause morbidity. Surgical complication rates for ND have ranged from $7 \%$ to $38 \%$ in several series [11-14]. It remains uncertain if resection confers benefit in terms of improving rates of local control, progression-free and overall survival. Additionally, the rate and clinical impact of pathologically involved lymph nodes with this approach remain uncertain. While there are several exploratory series investigating the use of positron emission tomography (PET) to prognosticate and select patients for ND $[15,16]$ this approach is not uniformly adopted, and utility remains uncertain. Finally, the impact of HPV status on rates of radiographic and pathologic residual disease, and on utility of ND, remains unknown.

This retrospective study examined a large cohort of patients with locally advanced SCCHN treated with definitive CRT and assessed the rate of radiographic residual disease (RA), the rate of pathologically positive residual disease, and underlying HPV status. The impact of RA presence and size on progression-free and overall survival were investigated.

\section{Materials and Methods}

This single institution, retrospective study involved a cohort of patients diagnosed with locally advanced SCCHN. The American Joint Committee on Cancer (AJCC) tumor, node, metastasis (TNM) staging was used to stage patients. Locally advanced SCCHN includes N1 to N3 disease. Patients with metastatic disease (M1), considered stage IVc, were excluded from the study. A query of the electronic medical record using ICD9 codes was performed to identify patients that met the inclusion criteria: age greater than 18 years, a histologically proven SCCHN diagnosed between 2003 and 2013, N1 to N3 disease at the time of diagnosis, frontline treatment with concurrent CRT (with or without induction chemotherapy), pre-treatment and post-treatment cross-sectional imaging to assess for response, available pathologic data (if elective ND was pursued), and any HPV status (positive, negative, or indeterminate). Each subject's unique identifiers were removed and replaced with an identification code. The Institutional review board (IRB) waived the requirement for patient consent, due to minimal patient risk (based on its retrospective nature), security of protected health information (PHI) (through de-identification), the lack of impact on particular patients' treatment, no additional need for new patient specimens, and the inclusion of deceased patients. Relevant data (including demographic, clinical, pathological, radiological, laboratory, and treatment-related) were abstracted through chart review and recorded by log.

\section{Statistical analysis}

Progression-free (PFS) and overall survival (OS) were estimated using Kaplan Meier analysis. Comparisons were made with the log rank test. Categorical variables were compared between subgroups using Fisher's exact test. Continuous variables were compared using the rank sum test. Logistic regression was used to perform a receiver operating characteristic analysis and to calculate the area under the ROC curve (AUC). All statistical analyses were conducted with SAS statistical software (SAS Institute Inc. 2012. SAS OnlineDoc ${ }^{\circledR}$ 9.4. Cary, NC: SAS Institute Inc.).

\section{Results}

Between January 2003 and December 2013, 123 patients diagnosed with locally advanced SCCHN, were evaluated at our institute. Of the 123 patients, analysis of complete information with regard to treatment and post therapy follow-up was available for ninety-nine patients. Patient characteristics are listed in Table 1.

Table 1 Patient demographics.

\begin{tabular}{|l|l|}
\hline 1) Age & \\
\hline median (range) & $56(31-74)$ \\
\hline 2) Gender & \\
\hline male & 74 \\
\hline
\end{tabular}




\begin{tabular}{|c|c|}
\hline female & 25 \\
\hline \multicolumn{2}{|l|}{ 3) Primary Site } \\
\hline Oropharynx & 77 \\
\hline Larynx & 9 \\
\hline Oral Cavity & 8 \\
\hline Other site & 5 \\
\hline \multicolumn{2}{|l|}{ 4) Tumor Stage } \\
\hline \multicolumn{2}{|l|}{ T stage } \\
\hline $\mathrm{T} 1$ & 26 \\
\hline T2 & 34 \\
\hline T3 & 16 \\
\hline $\mathrm{T} 4$ & 14 \\
\hline T unknown & 4 \\
\hline \multicolumn{2}{|l|}{$\mathrm{N}$ stage } \\
\hline $\mathrm{N} 1$ & 8 \\
\hline N2 & 86 \\
\hline N3 & 4 \\
\hline \multicolumn{2}{|l|}{ M stage } \\
\hline M1 & 0 \\
\hline \multicolumn{2}{|l|}{ 5) Race } \\
\hline African-American & 14 \\
\hline Asian/Pacific Islander & 2 \\
\hline Caucasian & 81 \\
\hline Latino & 1 \\
\hline Unknown & 1 \\
\hline \multicolumn{2}{|l|}{ 6) Tobacco } \\
\hline none & 30 \\
\hline $1-10$ years & 23 \\
\hline
\end{tabular}

\begin{tabular}{|l|l|}
\hline $11-20$ years & 12 \\
\hline $21-30$ years & 10 \\
\hline $31-40$ years & 11 \\
\hline $41+$ years & 13 \\
\hline 7) Alcohol use & \\
\hline yes & 32 \\
\hline no & 67 \\
\hline 8) p16 status & 37 \\
\hline yes & 11 \\
\hline no & 51 \\
\hline unknown & 35 months (4.4-90 months) \\
\hline 9) Follow-up time & \\
\hline median (range) & \\
\hline
\end{tabular}

Of the 99 patients treated, 35 underwent induction chemotherapy prior to CRT. Chemotherapy regimens for the 99 patients are listed in Table 2. All post-CRT neck imaging was executed with computed tomography. Following completion of CRT, 51/99 (52\%) patients were found to have radiologic RA. All patients who were found to have RA without distant metastatic disease on post-CRT imaging were referred for postCRT neck dissection. The median size of lymph nodes in those with radiologic RA was $1.4 \mathrm{~cm}$. Among patients with RA, 41/51 (80\%) underwent a post-CRT neck dissection. Of the 10 patients who did not undergo post-CRT ND for RA, 1 patient was found to have metastatic disease to the lungs at first follow-up CT scan. 4 patients refused surgery after it was recommended for persistent adenopathy, and 5 patients were lost to follow-up. Of those who underwent a post-CRT neck dissection, 8 (19.5\%) were found to have squamous cell carcinoma on pathology. Patients with RA who declined postCRT ND did not undergo fine needle aspiration (FNA) to confirm the presence of persistent disease.

Table 2 Chemotherapy regimens.

\begin{tabular}{|c|c|}
\hline \multicolumn{2}{|l|}{ 1) Induction chemotherapy } \\
\hline- Yes & 35 \\
\hline- No & 64 \\
\hline \multicolumn{2}{|l|}{ 2) Chemotherapy regimen with concurrent radiation } \\
\hline -Cisplatin $100 \mathrm{mg} / \mathrm{m}^{2} \mathrm{IV}$ days 1,22 and 43 & 51 \\
\hline -Cisplatin $40 \mathrm{mg} / \mathrm{m}^{2} \mathrm{IV}$ weekly & 6 \\
\hline \multicolumn{2}{|c|}{-Docetaxel $25 \mathrm{mg} / \mathrm{m}^{2} \mathrm{IV}, 5$-fluorouracil $60019 \mathrm{mg} / \mathrm{m}^{2} \mathrm{IV}$, hydroxyurea $500 \mathrm{mg}$ PO BID, days 1-5 followed by a 9 day break, 14 day cycle } \\
\hline -Carboplatin $\left(100 \mathrm{mg} / \mathrm{m}^{2}\right) /$ Paclitaxel $\left(45 \mathrm{mg} / \mathrm{m}^{2}\right), 6$ weekly & 6 \\
\hline -Carboplatin $\left(70 \mathrm{mg} / \mathrm{m}^{2}\right) / 5$-fluorouracil $\left(600 \mathrm{mg} / \mathrm{m}^{2}\right)$, IV, days $1-5,29-33$ & 2 \\
\hline -Carboplatin $\left(100 \mathrm{mg} / \mathrm{m}^{2}\right)$ weekly & 11 \\
\hline
\end{tabular}


At a median follow-up of 35 months (range: 4.4-90), 7 deaths and 18 recurrences were noted amongst all patients. There was no difference in overall survival (4 deaths amongst patients with RA vs. 3 in patients without RA; $p=0.72$ ) or progression free survival (9 recurrences amongst patients with RA vs. 8 in patients without RA; $p=0.37$ ) between patients found to have post-CRT RA on CT scan compared to those without RA (Figure 1).

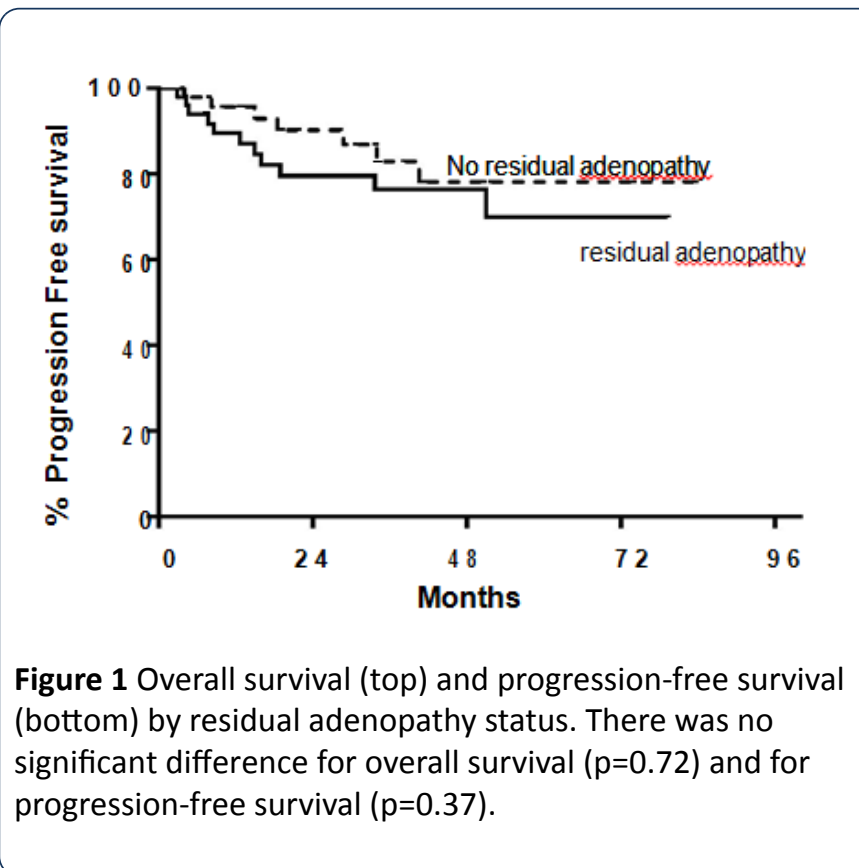

Of the 7 deaths recorded amongst all patients, 4 occurred in those with residual adenopathy on CT and 3 occurred in those patients without residual adenopathy. Of 17 the diameter of radiologic $R A$ ( $A U C=0.64$ ) was not predictive of pathologic residual cancer $(p=0.50)$. Although $p 16$ status was not available for several patients in our analysis, p16 positivity was statistically significant for improved overall survival $(p=0.038)$. Overall survival at 1,2 and 5 years amongst p16 positive patients was noted to be $100 \%$ compared to $88 \%, 73 \%$ and $73 \%$ amongst those with p16 negativity, respectively. P16 positive status did not have a statistically significant impact on progression free survival $(p=0.27)$. Several factors were evaluated in a univariate analysis to ascertain their impact on pathologic RA. None was found to predict for pathologic RA disease ( $p 16: p=0.36$, alcohol use: $p=0.71, T$-stage: $p=0.65, N$ stage: $p=0.99$, tobacco use: $p=0.57$ ). Size of radiographic residual disease was related to residual cancer present on pathologic analysis by logistic regression with $p=0.50$. The best cutpoint for predicting pathologic residual disease was $\geq 0.9$ $\mathrm{cm}$ with a sensitivity of $100 \%$ and specificity of $53 \%$ (Figure 2; Table 3).

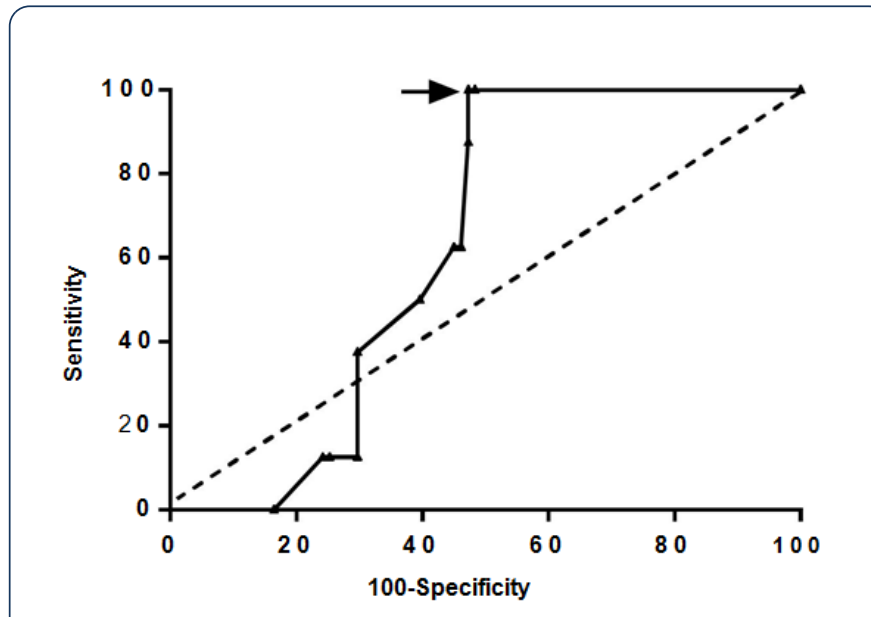

Figure 2 Receiver operating curve relating size of radiographic residual disease to residual cancer present on pathologic analysis ( $A \cup C=0.64)$. Arrow indicates the cutpoint of $\geq 0.9 \mathrm{~cm}$ to predict pathologic residual cancer.

Table 3 Predictive factors for residual adenopathy.

\begin{tabular}{|l|l|}
\hline Predictive factor & p-value \\
\hline P16 status & 0.36 \\
\hline Alcohol usage & 0.71 \\
\hline T-stage & 0.65 \\
\hline N-stage & 0.99 \\
\hline Tobacco usage & 0.57 \\
\hline
\end{tabular}

\section{Discussion}

In this retrospective, single institution review, we have demonstrated that the usage of post CRT RA is a poor predictor for residual pathologic disease in the neck. CT scan accurately predicted residual disease in only $19.5 \%$ of the patients in this analysis. More than $80 \%$ of patients therefore underwent a post-CRT neck dissection, without evidence of malignancy by pathology, which exposed them to additional morbidity without any benefit to local disease control, progression free survival or overall survival. Furthermore, the diameter of radiologic RA did not predict pathologic residual cancer, in our patient cohort. Our study demonstrated that the use of post-CRT CT imaging is not an efficacious tool for determining which patients will benefit from ND. Our analysis demonstrated that several factors implicated in SCCHN, including alcohol and tobacco exposure, were not predictive of pathologic residual disease, despite their known impact on head and neck tumorigenesis [17]. Although advanced TNM staging is well known to adversely impact prognosis in patients with SCCHN, it did not predict the presence of residual disease in our cohort. These data demonstrate the need for an 
effective, reliable biomarker and/or imaging modality to accurately stratify patients to ND following CRT. These data confirm that most patients who complete CRT may forego post-CRT ND.

The limits of our study are related to its retrospective nature. Furthermore, unequal representation of nodal stages, sites of primary malignancy, chemotherapy regimens, and degrees of follow up care may impact the rates and outcomes of SCCHN. Not all patients with RA elected to undergo ND (41 patients underwent ND of the 51 patients found to have RT on CT scan). It remains unknown whether the remaining 10 patients would have been found to have residual cancer on pathologic evaluation. Despite limitations, these findings suggest many patients can safely forego post-treatment ND despite RA.

The limitation of radiologic RA as a prognostic indicator has been explored in the literature. In Porceddu et al. analysis of the utility of FDG-PET in the post-CRT setting, the positive predictive value of CT was found to be only $14 \%$ [18], similar to the value of RA noted in our patients who underwent ND. Other studies, however, have found a different rate of concordance between $\mathrm{CT}$ nodal abnormalities and findings in the ND specimen found in other series $[19,20]$. Forest et al. conducted a retrospective, single institution review of the management of patients [2] with N2-N3 disease following CRT. They observed a $68 \%$ regional response rate amongst all patients (regional CR, defined as a greater than $90 \%$ reduction in the volume of the lymph node and/or reduction to less than $1 \mathrm{~cm}$ and be oval-shaped with smooth borders with without imaging evidence of extra-capsular spread, necrosis or enhancing areas). Amongst those patients who achieved a regional $C R$, there was an overall recurrence rate of $5 \%$. Patients who achieved a regional partial response (regional PR, defined as anything less than a regional $C R$ ) then underwent ND following completion of CRT. $40 \%$ of patients were found to have residual disease on pathology. Whether the presence of viable tumor cells (VTCs) has prognostic significance is also unclear. If RA, as that observed in the Porceddu study as well as our own, suggests the presence of VTCs, this may imply that VTCs have low proliferative potential, and is further supported by the low positive predictive value of CT scans [21]. However, Ganly et al. reported that patients with VTCs in postchemo radiation neck dissection specimens had a poorer outcome compared to those patients with no VTCs [22]. These conflicting observations indicate that additional research is needed on the role of viable tumor cells and their implications of SCCHN patients with advanced nodal disease.

Clinical data regarding the management of patients with complete clinical response supports the role of surveillance over a neck dissection. Low rates of isolated neck recurrences (0-14\%) have been observed in patients achieving a clinical complete response (cCR) [23]. Additionally, historical data has not supported the notion that planned ND improves regional control or overall survival in patients with a cCR [24,25]. Given the lack of improved PFS and OS and the potential for significant morbidity associated with the procedure, physicians have increasingly adopted observation over PND in those patients who achieve a cCR. The management of patients with a partial clinical response (cPR) continues to be a subject of considerable debate. Soltys et al. reviewed the role of a planned neck dissection (PND) after sequential chemoradiotherapy in patients with SCCHN and N2-N3 disease [20]. In their retrospective analysis, they reviewed 90 patients with N2-N3 disease treated at a single institution over a 10 year period. Patients who completed two prospective organ preservation sequential CRT protocols (OSP2 and OSP3) were then assessed with physical examination, direct fiberoptic evaluation and CT or MRI. Those who had a complete clinical response, defined as no palpable lymph nodes in the neck and no lymph nodes $-1 \mathrm{~cm}$ did not undergo a partial neck dissection following completion of CRT. Those with a $\mathrm{CCR}$ to the primary site but a clinical partial response (CPR) to the neck (defined as anything less than a CCR) underwent a planned ND. Of the 56 patients (62\%) who achieved a cCR in the neck, $13(23 \%)$ had experienced a relapse at the conclusion of the study. Of the 5 patients with neck failures, only 2 (one with neck recurrence, one patient with neck and distant recurrence) were felt to have benefitted from a post-CRT ND ( 2 of 48 patients, or $2 \%$ ). 34 patients (38\%) had a cPR in the neck, 30 of whom underwent a ND. Amongst these patients, the $\mathrm{PCR}$ rate was $53 \%(n=16)$. Of those patients found to have a pCR, 2 (13\%) experienced disease relapse. Those patients with pPR fared worse, with $7 / 14$ patients (50\%) experiencing disease recurrence. These data, unlike those presented in our analysis, would suggest that patients with a partial clinical response should undergo a post-CRT neck dissection, owing to the high risk of relapsed disease. This study, as is our own, is limited by its retrospective nature and limitation to a single institution.

The utilization of PET/CT imaging in the surveillance of SCCHN patients following CRT has emerged as a preferred modality in the prognostic and therapeutic stratification of patients. PET/CT may assist in more precisely delineating those patients for whom ND is not necessary. Investigations have drawn disparate conclusions about whether FDG avidity accurately predicts the presence of residual active cancer $[26,27]$. There are important limitations that must be considered with these data, however. First, the resolution of nascent forms of FDG-PET was considerably lower than that available with contemporary scanners. Furthermore, several early studies, such as those by Rogers et al. employed a subjective numerical scale, rather than the standardized uptake value (SUV) utilized today. Additionally, the timing of PET-CT in the post-CRT setting is important to appropriately delineate its positive and negative predictive value, with a PET/CT scan performed 8-12 weeks after the completion of CRT considered the optimal window $[15,28,29]$

While prior studies showed data supporting the continued necessity of ND in the setting of PET negative RA [26] there is ample supportive evidence showing that clinical absence of adenopathy and negative PET accurately predict the absence of residual active disease, allowing omission of ND $[15,16,29,30]$. Yao et al. retrospective analysis found that PETCT conducted 12 weeks after completion of CRT had a negative predictive value of $100 \%$ and a positive predictive value of $43 \%$ [30]. Another retrospective analysis demonstrated a similarly 
high NPV in the setting of normal FDG-PET/CT $\geq 8$ weeks after completion of CRT, regardless of the presence of RA (NPV: 94-98\%) [15]. Porceddu et al. conducted a prospective study to evaluate the role of FDG-PET/CT in node positive SCCHN patients who received definitive RT with or without chemotherapy. 112 patients who achieved a complete response at the primary site then underwent screening with CT (+/-MRI)+PET-CT. 50/112 patients (45\%) were found to have RA (defined as $-1 \mathrm{~cm}$ ). 41 of those patients had a negative $\mathrm{PET} / \mathrm{CT}$ and did not undergo ND. With a median follow-up time of 28 months, all patients with a negative $\mathrm{PET} / \mathrm{CT}$ remained free of nodal failure at last follow-up. The NPVs for PET and CT nodal response assessment were $98.1 \%$ (95\% Cl, 93.2\%-99.8\%) and $96.1 \%$ (95\% Cl, 88.8\%-99.6\%), respectively. The PPVs for PET and CT were $77.8 \%(95 \% \mathrm{Cl}, 40 \%-97.2 \%)$ and $14 \%(95 \% \mathrm{Cl}$, $5.8 \%-26.7 \%)$, respectively. This emerging body of data suggests that PET-CT has a high NPV for post-CRT patients. There is conflicting data on the positive predictive value of PET-CT. Additional prospective data is needed to clarify the role of FDG-PET/CT in the management of SCCHN with advanced nodal disease.

\section{Conclusions}

Following CRT, while many patients have RA, only a small subset $(19.5 \%)$ have residual active cancer. The presence of either does not impact PFS or OS. Moreover, using RA greater than $1 \mathrm{~cm}$ as a threshold to perform an ND lacks specificity in identifying those with pathologically positive residual cancer. These data suggest ND may be safely avoided in most patients and will help inform future prospective trials using biomarkers and/or alternative imaging modalities, such as PET/CT, to identify the subset of patients most likely to benefit from ND.

\section{References}

1. Siegel R, Naishadham D, Jemal A (2013) Cancer statistics. CA: Cancer J Clin 63: 11-30.

2. Chaturvedi AK, Engels EA, Pfeiffer RM, Hernandez BY, Xiao W, et al. (2011) Human papillomavirus and rising oropharyngeal cancer incidence in the United States. J Clin Oncol 29: 4294-4301.

3. Mehanna H, Beech T, Nicholson T, El-Hariry I, McConkey C, et al. (2013) Prevalence of human papillomavirus in oropharyngeal and nonoropharyngeal head and neck cancer--systematic review and meta-analysis of trends by time and region. Head Neck 35: 747-55.

4. The Department of Veterans Affairs Laryngeal Cancer Study Group (1991) Induction chemotherapy plus radiation compared with surgery plus radiation in patients with advanced laryngeal cancer. N Engl J Med 324: 1685-1690.

5. Spaulding MB, Fischer SG, Wolf GT (1994) Tumor response, toxicity, and survival after neoadjuvant organ-preserving chemotherapy for advanced laryngeal carcinoma. The Department of Veterans Affairs Cooperative Laryngeal Cancer Study Group. J Clin Oncol 12: 1592-1599.

6. Pignon JP, le Maître A, Maillard E, Bourhis J, MACH-NC Collaborative Group (2009) Meta-analysis of chemotherapy in head and neck cancer (MACH-NC): an update on 93 randomised trials and 17,346 patients. Radiother Oncol 92: 4-14.

7. Pfister DG, Spencer S, Brizel DM, Burtness B, Busse PM, et al. (2014) Head and neck cancers. Version 2.2014. Clinical practice guidelines in oncology. J Natl Compr Canc Netw 12: 1454-1487.

8. Ang KK, Harris J, Wheeler R, Weber R, Rosenthal DI, et al. (2010) Human papillomavirus and survival of patients with oropharyngeal cancer. N Engl J Med 363: 24-35.

9. Rischin D, Young RJ, Fisher R, Fox SB, Le QT, et al. (2010) Prognostic significance of p16INK4A and human papillomavirus in patients with oropharyngeal cancer treated on TROG 02.02 phase III trial. J Clin Oncol 28: 4142-4148.

10. Lavertu P, Adelstein DJ, Saxton JP, Secic M, Wanamaker JR, et al. (1997) Management of the neck in a randomized trial comparing concurrent chemotherapy and radiotherapy with radiotherapy alone in resectable stage III and IV squamous cell head and neck cancer. Head Neck 19: 559-566.

11. Brizel DM, Prosnitz RG, Hunter S, Fisher SR, Clough RL, et al. (2004) Necessity for adjuvant neck dissection in setting of concurrent chemoradiation for advanced head-and-neck cancer. Int J Radiat Oncol Biol Phys 58: 1418-1423.

12. Narayan K, Crane CH, Kleid S, Hughes PG, Peters LJ (1999) Planned neck dissection as an adjunct to the management of patients with advanced neck disease treated with definitive radiotherapy: for some or for all? Head Neck 21: 606-613.

13. Davidson BJ, Newkirk KA, Harter KW, Picken CA, Cullen KJ, et al. (1999) Complications from planned, posttreatment neck dissections. Arch Otolaryngol Head Neck Surg 125: 401-405.

14. Lavertu P, Bonafede JP, Adelstein DJ, Saxton JP, Strome M, et al. (1998) Comparison of surgical complications after organpreservation therapy in patients with stage III or IV squamous cell head and neck cancer. Arch Otolaryngol Head Neck Surg 124: 401-406.

15. Ong SC, Schöder H, Lee NY, Patel SG, Carlson D, et al. (2008) Clinical utility of 18F-FDG PET/CT in assessing the neck after concurrent chemoradiotherapy for Locoregional advanced head and neck cancer. J Nucl Med 49: 532-540.

16. Nayak JV, Walvekar RR, Andrade RS, Daamen N, Lai SY, et al. (2007) Deferring planned neck dissection following chemoradiation for stage IV head and neck cancer: the utility of PET-CT. Laryngoscope 117: 2129-2134.

17. Dal Maso L, Torelli N, Biancotto E, Di Maso M, Gini A, et al. (2015) Combined effect of tobacco smoking and alcohol drinking in the risk of head and neck cancers: a re-analysis of casecontrol studies using bi-dimensional spline models. Eur J Epidemiol.

18. Porceddu SV, Pryor DI, Burmeister E, Burmeister BH, Poulsen MG, et al. (2011) Results of a prospective study of positron emission tomography-directed management of residual nodal abnormalities in node-positive head and neck cancer after definitive radiotherapy with or without systemic therapy. Head Neck 33: 1675-1682.

19. Forest VI, Nguyen-Tan PF, Tabet JC, Olivier MJ, Larochelle D, et al. (2006) Role of neck dissection following concurrent chemoradiation for advanced head and neck carcinoma. Head Neck 28: 1099-105.

20. Soltys SG, Choi CY, Fee WE, Pinto HA, Le QT (2012) A planned neck dissection is not necessary in all patients with N2-3 head- 
and-neck cancer after sequential chemoradiotherapy. Int J Radiat Oncol Biol Phys 83: 994-999.

21. Da Mosto MC, Lupato V, Romeo S, Spinato G, Addonisio G, et al. (2013) Is neck dissection necessary after induction plus concurrent chemoradiotherapy in complete responder head and neck cancer patients with pretherapy advanced nodal disease? Ann Surg Oncol 20: 250-256.

22. Ganly I, Bocker J, Carlson DL, D’Arpa S, Coleman M, et al. (2011) Viable tumor in postchemoradiation neck dissection specimens as an indicator of poor outcome. Head Neck 33: 1387-1393.

23. Wee JT, Anderson BO, Corry J, D'Cruz A, Soo KC, et al. (2009) Management of the neck after chemoradiotherapy for head and neck cancers in Asia: consensus statement from the Asian Oncology Summit 2009. Lancet Oncol 10: 1086-1092.

24. Argiris A, Stenson KM, Brockstein BE, Mittal BB, Pelzer $H$, et al. (2004) Neck dissection in the combined-modality therapy of patients with locoregionally advanced head and neck cancer. Head Neck 26: 447-455.

25. Liauw SL, Mancuso AA, Amdur RJ, Morris CG, Villaret DB, et al. (2006) Postradiotherapy neck dissection for lymph node-positive head and neck cancer: the use of computed tomography to manage the neck. J Clin Oncol 24: 1421-1427.
26. Rogers JW, Greven KM, McGuirt WF, Keyes JW Jr, Williams DW, et al. (2004) Can post-RT neck dissection be omitted for patients with head-and-neck cancer who have a negative PET scan after definitive radiation therapy? Int J Radiat Oncol Biol Phys 58: 694-697.

27. Brkovich VS, Miller FR, Karnad AB, Hussey DH, McGuff HS, et al. (2006) The role of positron emission tomography scans in the management of the $\mathrm{N}$-positive neck in head and neck squamous cell carcinoma after chemoradiotherapy. Laryngoscope 116 : 855-858.

28. Yao M, Graham MM, Hoffman HT, Smith RB, Funk GF, et al. (2004) The role of post-radiation therapy FDG PET in prediction of necessity for post-radiation therapy neck dissection in locally advanced head-and-neck squamous cell carcinoma. Int J Radiat Oncol Biol Phys 59: 1001-1010.

29. Porceddu SV, Jarmolowski E, Hicks RJ, Ware R, Weih L, et al. (2005) Utility of positron emission tomography for the detection of disease in residual neck nodes after (chemo)radiotherapy in head and neck cancer. Head Neck 27: 175-181.

30. Yao M, Smith RB, Graham MM, Hoffman HT, Tan H, et al. (2005) The role of FDG PET in management of neck metastasis from head-and-neck cancer after definitive radiation treatment. Int J Radiat Oncol Biol Phys 63: 991-999. 